\title{
Evaluation of fluoride bioavailability in toothpastes
}

\author{
Hae-Youn Ko ${ }^{1,2}$, Si-Mook Kang ${ }^{1,2}$, Ho-Keun Kwon ${ }^{1,2}$, Baek-II Kim ${ }^{1,2,3}$ \\ 'Department of Preventive Dentistry \& Public Oral Health, ${ }^{2}$ BK21 PLUS Project, ${ }^{3}$ Oral Science Research Institute, \\ Yonsei University College of Dentistry, Seoul, Korea
}

Received: January 14, 2015

Revised: April 5, 2015

Accepted: April 20, 2015

\section{치약 내 불소의 생리적 가용능 평가}

\author{
고혜연 ${ }^{1,2}$, 강시묵 ${ }^{1,2}$, 권호근 ${ }^{1,2}$, 김백일 ${ }^{1,2,3}$ \\ '연세대학교 치과대학 예방치과학교실, ${ }^{2} \mathrm{BK} 21$ 통합구강생명사업단, ${ }^{3}$ 구강과학연구소
}

Corresponding Author: Baek-\| Kim Department of Preventive Dentistry \& Public Oral Health, Yonsei University College of dentistry, 50 Yonsei-ro, Seoul 120-749, Korea

Tel: +82-2-2228-3070

Fax: $+82-2-392-2926$

E-mail: drkbi@yuhs.ac
Objectives: The aims of this study were to determine the total fluoride concentration and bioavailable fluoride concentration in different toothpastes, based on a newly suggested method by the International Organization for Standardization (ISO), and to compare the measured concentrations with the concentrations written on the packaging.

Methods: The concentrations of total fluoride (TF) and bioavailable fluoride (BF) were measured in six toothpastes. For the TF measurement, $1 \mathrm{~g}$ of each toothpaste was mixed with dipotassium hydrogen phosphate $\left(\mathrm{K}_{2} \mathrm{HPO}_{4}\right)$, and hydrogen chloride $(\mathrm{HCl})$ was placed. After 24 hours, the samples were centrifuged and total ionic strength adjustment buffer (TISAB) solution was added. For the BF measurement, the toothpaste was mixed with $\mathrm{K}_{2} \mathrm{HPO}_{4}$ for only 1 minute. The samples were centrifuged, and then $\mathrm{HCl}$ was placed and allowed to stand for 24 hours. The TISAB solution was added subsequently. The concentration of fluoride ions was measured using a fluoride ion-selective electrode and calculated against a standard curve.

Results: The six toothpastes were composed of different fluoride compounds and abrasives. The measured TF concentration ranged from $624.99 \mathrm{ppm}$ to $1,353.00 \mathrm{ppm}$, and the similarity to the declared fluoride concentration ranged from $53.48 \%$ to $93.31 \%$. The measured BF concentration ranged from $587.61 \mathrm{ppm}$ to $1,360.05 \mathrm{ppm}$, and the similarity to the expected fluoride concentration ranged from $41.97 \%$ to $93.80 \%$. Two samples were clearly separated when the samples were centrifuged, whereas the remaining four samples had unclear supernatants. The clearly separated toothpastes (i.e., toothpastes 5 and 6) had BF concentrations that were similar to or lower than the declared fluoride concentrations and the measured TF concentrations. However, the unclearly separated toothpastes showed inconsistent relationships between the measured TF and BF concentrations.

Conclusions: The measured TF and BF concentrations of the six toothpastes did not reach the expected fluoride concentration. This finding resulted from the different compositions and forms of the toothpastes. Therefore, the properties of toothpastes need to be considered when measuring their fluoride concentrations.

Key Words: Fluoride, Toothpaste, Bioavailability, Standards 


\section{Introduction}

The large reduction in number of dental caries in the last few decades is considered to be due to the widespread use of both systemic and topical fluorides ${ }^{1}$. As the beneficial effect of topical fluoride has been proven through number of studies ${ }^{2,3)}$, there was a huge improvement on the topical fluorides, with toothpaste still remaining as the most widespread and significant form of fluoride ${ }^{4,5}$. There was a Cochrane systematic review that also emphasized the role of fluoride toothpaste in anti-caries activity, yet it stated that the concentration of fluoride in toothpastes needs to be 1,000 ppm or more in order to be effective ${ }^{6}$.

On the other hand, some of early studies on the fluoride toothpastes failed to prove the efficacy of the toothpastes against caries without finding the exact mechanism ${ }^{7,8)}$. However, as more number of investigations were done on fluoride toothpastes, it was found that for the toothpastes to be effective against caries, the fluorides need to be in soluble form and that the anti-caries effect could be reduced by insoluble salts formed between the free fluoride ions and the abrasives contained in the toothpastes ${ }^{9,10)}$. Thus, it is crucial for the toothpastes to contain sufficient concentration of not only the total fluoride but also the soluble fluoride to be effective in prevention of caries.

However, the concentration of soluble fluoride can be lower than that of the total fluoride which is labeled on the packaging. In Korea, there were several attempts to measure the total fluoride and the soluble fluoride of the commercialized toothpastes in Korea, and they found that the majority of the analyzed toothpastes contained soluble fluoride close to $1,000 \mathrm{ppm}^{11,12}$. Considering the maximum permitted fluoride content in toothpaste used to be 1,000 ppm when these studies were performed, it can be assumed that most of the fluoride toothpastes commercialized in Korea contained soluble fluoride in close proximity of the labeled fluoride. Nevertheless, there was a previous study which determined total and free fluoride from the toothpastes purchased in low-income countries, and they reported that in $25 \%$ of the toothpastes, $<55 \%$ of the declared fluoride was in free fluoride form ${ }^{10}$. Therefore, in order to ensure the efficacy of fluoride toothpastes, there is a need of quality control measures that test toothpastes by measuring both total fluoride and soluble fluoride.

There are various measurement methods for fluoride in toothpastes. As one of the examples, International Organization for Standardization (ISO), who defines standards for the quality of fluoride toothpaste, describes methods for testing the total fluoride content of toothpastes in its standard $11609^{13)}$, yet the guideline for soluble fluoride is still in preparation. Recently, a suggestion for measuring the soluble fluoride was made by the ISO TC 106. The suggested method measures the concentration of soluble fluoride in toothpaste slurry at one minute. As the actual tooth brushing time has been known to be approximately 1 minute ${ }^{14,15)}$, this method can be considered as the representative method to capture all the bioavailable fluoride species during the 1-minute brushing. However, the fluoride measurement method often used in Korea is the diffusion method, which measures soluble fluoride concentration without considering the actual brushing time. Therefore, there is a need to evaluate the 1-minute fluoride bioavailability also in Korea.

The aims of this study were to establish the total and 1-minute potentially available soluble fluoride concentration of toothpastes that were available in many countries using the newly suggested method and to compare the concentrations with the concentration written on the packaging.

\section{Materials and Methods}

\section{Fluoride toothpaste samples}

Six toothpastes of five brands commercialized in many countries were evaluated (Table 1). Along with the instruction sheet information about the toothpastes such as lot numbers, density and concentrations of fluoride compounds, and expiration dates were provided by the ISO TC 106. The information provided on packaging was also checked for descriptive names of fluoride compounds and abrasives.

\section{Preparation of the samples}

Two different fluoride concentrations were assessed for each toothpaste: (1) Total fluoride and (2) 1-minute bioavailable fluoride. For both assessments, approximately $1 \mathrm{~g}$ of the toothpaste was measured into a tared test tube, and mixed with $0.1 \mathrm{~mol} / \mathrm{L} \mathrm{K}_{2} \mathrm{HPO}_{4}$ (Duksan, Ansan, Korea) at a 1:3 dilution to create a slurry sample. For each toothpaste, 2 slurry samples were produced; one for determination of Total Fluoride and the other for determination of Bioavailable Fluoride. After 12 slurry samples were produced from six toothpastes, the fluoride preparation was carried on as follows.

\subsection{Determination of Total Fluoride (TF)}

The six slurry samples were thoroughly vortexed, and 1.0 $\mathrm{mol} / \mathrm{L} \mathrm{HCl}$ of the twice the slurry volume was added to each 
Table 1. Information of toothpastes under analysis

\begin{tabular}{lcccc}
\hline $\begin{array}{c}\text { Commercial brand } \\
\text { (Manufacturer) }\end{array}$ & Code & $\begin{array}{c}\text { F } \\
\text { compound }\end{array}$ & $\begin{array}{c}\text { Expected } \\
{[\mathrm{F}](\mathrm{ppm})}\end{array}$ & Abrasive \\
\hline Cavity Protection (Colgate) & 1 & $\mathrm{SMFP}$ & 1,000 & Dicalcium phosphate dihydrate \\
Elmex (Gaba) & 2 & $\mathrm{AmF}$ & 1,400 & Hydrated silica \\
Elmex Erosion (Gaba) & 3 & $\mathrm{AmF}$ & 1,400 & Hydrated silica \\
& & $\mathrm{NaF}$ & 1,100 & Hydrated silica \\
Cavity Protection (Crest) & 4 & $\mathrm{NaF}$ & 1,100 & Silica \\
Complete Care (Arm \& Hammer) & 5 & $\mathrm{NaF}$ & 1,450 & Calcium glycerophosphate \\
Fluro Protector Gel (Ivoclarvivadent) & 6 & $\mathrm{KF}$ & & \\
\hline
\end{tabular}

SMFP, sodium monofluorophosphate; AmF, amine fluoride; NaF, sodium fluoride; KF, potassium fluoride.

Table 2. Concentrations of expected fluoride, measured total fluoride, and measured bioavailable fluoride of the toothpastes (Mean \pm SD)

\begin{tabular}{cccrc}
\hline Toothpastes & $\begin{array}{c}\text { F } \\
\text { compound }\end{array}$ & $\begin{array}{c}\text { Expected } \\
{[\mathrm{F}]} \\
(\mathrm{ppm})\end{array}$ & $\begin{array}{c}\text { Measured } \\
\text { Total }[\mathrm{F}] \\
(\mathrm{ppm})(\mathrm{n}=1)\end{array}$ & $\begin{array}{c}\text { Measured } \\
\text { Bioavailable }[\mathrm{F}] \\
(\mathrm{ppm})(\mathrm{n}=1)\end{array}$ \\
\hline 1 & $\mathrm{SMFP}$ & 1,000 & $744.92 \pm 126.95$ & $720.62 \pm 44.75$ \\
2 & $\mathrm{AmF}$ & 1,400 & $748.75 \pm 107.24$ & $1,100.74 \pm 62.98$ \\
3 & $\mathrm{AmF}$ & 1,400 & $1,048.95 \pm 77.07$ & $587.61 \pm 62.07$ \\
& $\mathrm{NaF}$ & 1,100 & $624.99 \pm 23.49$ & $945.22 \pm 39.08$ \\
5 & $\mathrm{NaF}$ & 1,100 & $1,017.29 \pm 103.76$ & $835.97 \pm 61.83$ \\
6 & $\mathrm{NaF}$ & 1,450 & $1,353.00 \pm 115.16$ & $1,360.05 \pm 103.88$ \\
\hline
\end{tabular}

SMFP, sodium monofluorophosphate; AmF, amine fluoride; NaF, sodium fluoride; KF, potassium fluoride.

test tube in order to ionize fluoride ion. Acidified slurry was maintained for 24 hours followed by the transfer of the $1 \mathrm{ml}$ into a microcentrifuge tube. The sample was centrifuged (Micro 17R, Hanil Co., Inchon, Korea) at 12,000 rpm for 10 minutes. After the centrifuge, $0.5 \mathrm{ml}$ of the supernatant was transferred into another microcentrifuge tube, and it was followed by addition of the same volume of TISAB solution, which was TISAB II (trans-1,2-Diaminocyclohexane- $N, N, N^{\prime}, N^{\prime}$-tetraacetic acid, Orion Research Inc. USA) diluted with deionized water at 1:1 ratio in order to prevent interference from other ions.

\subsection{Determination of Bioavailable Fluoride (BF)}

To obtain bioavailable fluoride, the six slurry samples were mixed by alternating between high speed vortexing and manual mixing for 1 minute only. If the toothpaste was not completely dispersed, it was discarded, and the procedure was repeated. $0.5 \mathrm{ml}$ slurry was then transferred into a microcentrifuge tube and centrifuged (Micro 17R, Hanil Co., Inchon, Korea) at 12,000 rpm for 2 minutes. $0.1 \mathrm{ml}$ of the supernatant after the centrifuge was mixed with $0.1 \mathrm{ml} 2.0 \mathrm{~mol} / \mathrm{L} \mathrm{HCl}$ and digested overnight. After overnight, $0.3 \mathrm{ml}$ TISAB solution was added to the digested sample and mixed thoroughly.

\section{Fluoride analysis}

To determine both TF and BF concentrations, $4,950 \mathrm{ml}$ TISAB solution was added to $0.05 \mathrm{ml}$ of each sample of all twelve samples. For measurements, a fluoride electrode (Orion Research Inc., ion plus fluoride 9609BN, USA) was used, which was calibrated with $\mathrm{NaF}$ standard solutions of $1 \times 10^{-2}, 1 \times 10^{-3}$, $1 \times 10^{-4}$, and $1 \times 10^{-5} \mathrm{~mol} / \mathrm{L}$ made with $\mathrm{NaF}$ (Sigma Chemical Co., St. Louis, Mo., USA). The Fluoride concentrations were calculated using a calibration curve based on the standard solutions.

\section{Results}

The results of the fluoride analysis of the six toothpastes are presented in Table 2. The evaluated toothpastes had various types of fluorides and abrasives. Among the six toothpastes evaluated, there were two toothpastes only with $\mathrm{NaF}$, one only with $\mathrm{AmF}$, and one with a combination of $\mathrm{NaF}$ and AmF. The other two toothpastes had SMFP and KF as fluoride agents respectively (Table 1). All the TF concentrations were lower than the fluoride contents declared by the manufacturer. When the TF concentrations were compared to the expected fluoride concentrations, the percentages of 


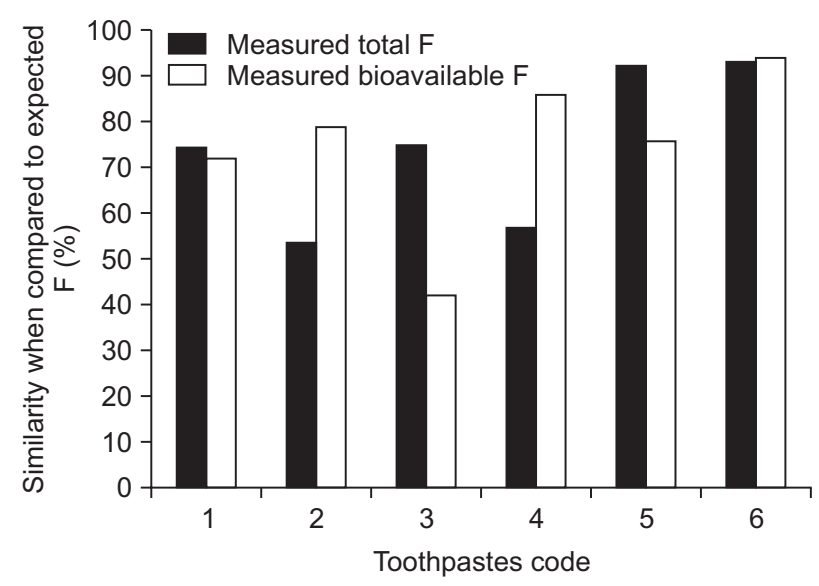

Fig. 1. Percentages of similarities between the expected fluoride and the measured total fluoride (dark bars) and also between the expected fluoride and the measured bioavailable fluoride (light bars) of the toothpastes.

similarities between them could be ranked in descending order as follows: Toothpaste 6 (93.31\%), toothpaste 5 (92.48\%), toothpaste $3(74.93 \%)$, toothpaste $1(74.49 \%)$, toothpaste 4 (56.82\%), toothpaste 2 (53.48\%) (Fig. 1).

Regarding the $\mathrm{BF}$ content, all the measured BF contents were also lower than the declared fluoride contents with the similarities also varying from $41.97 \%$ to $93.80 \%$ (Table 2 and Fig. 1). For BF concentration measurement, the toothpaste samples were centrifuged for 2 minutes, and the toothpastes could be divided into two groups depending on the status of resulting supernatant after the centrifuge. Among the six toothpastes, two toothpaste samples 5 and 6 were distinctively separated into supernatants and precipitates while the other four toothpaste slurry samples were not clearly separated, producing opaque supernatants. When the BF contents of the toothpastes 5 and 6 with clear separation were compared to the measured TF of those, the toothpaste 6 had similar fluoride contents for both measurements, whereas the BF of toothpaste 5 was $82.17 \%$ of the measured TF. The other toothpastes 1, 2, 3, and 4, which were not apparently separated after the centrifuge, were found to show inconsistent relations between the measured $\mathrm{TF}$ and $\mathrm{BF}$. Among the four samples, the $\mathrm{BF}$ concentrations of the toothpaste 1 and 3 were lower than the measured TF while the other two toothpastes 2 and 4 showed an opposite trend.

\section{Discussion}

Fluoride toothpastes which are widely used have a crucial role in caries prevention ${ }^{16}$. However, in order to interfere with the caries process, fluoride ions need to be soluble in their formula of the fluoride toothpastes so that they can be available for the de- and remineralization process ${ }^{10}$. However, other components of toothpaste including abrasives can bind to fluoride ions and interfere with their activity ${ }^{17)}$. Therefore, for anti-caries effects of fluoride toothpastes to be valid, the formulation has to be compatible ${ }^{18)}$. There has been number of attempts to find the ideal formulation of toothpaste and the appropriate measurement method to assess soluble fluoride ${ }^{19-21}$. However, even throughout the development of measurement methods, there is no standardized method to measure the soluble fluoride currently. The present study followed the ISO protocol to measure the total fluoride and another newly suggested protocol to measure the concentration of soluble fluoride in toothpaste slurry mixed at one minute, which represents the fluoride concentration potentially bioavailable to the toothpaste user during brushing.

The results of the present finding confirmed that of various factors associated with fluoride compatibility, fluoride agent and abrasive formulated in toothpaste are the ones that play a key role. Currently, most fluoride toothpastes contain either sodium fluoride (NaF) or sodium monofluorophosphate (SMFP) or a combination of both as fluoride agents. A previous study claimed the superiority of ionic compound $\mathrm{NaF}$, which can be easily dissociated, compared to the covalently bonded compound SMFP, which requires enzymatic hydrolysis to release fluoride ions ${ }^{22}$. In this study, the analyzed toothpastes also contained SMFP and NaF, and as well as potassium fluoride $(\mathrm{KF})$ and amine fluoride $(\mathrm{AmF})$. The superiority of ionic compound over covalent compound can also be supported by the finding of the present study where the toothpastes 5 and 6, which contained ionic fluoride compounds, had higher $\mathrm{TF}$ and $\mathrm{BF}$ concentrations than the toothpastes containing fluoride compounds based on covalent bonding. However, this finding could also be attributed to the abrasive, which is another factor associated with efficacy of toothpastes.

In the present study, the total fluoride was measured by ionizing the toothpaste with acid first, which was followed by the centrifugation, and compared with the declared fluoride concentration. The measured TF concentrations of all six toothpastes were lower than the declared TF concentrations with a range of differences, and the differences could be due to the reactions between the various fluoride compounds and abrasives of the six toothpastes. SMFP is known to be compatible with a wider range of abrasives than $\mathrm{NaF}$ because $\mathrm{NaF}$ is incompatible with abrasives that contain calcium; particularly calcium carbonate $\left(\mathrm{CaCO}_{3}\right)$ and dicalcium phosphate de- 
hydrate (DCPD). Its fluoride can easily dissociate due to ionic bonding, strongly react with these abrasives, and form insoluble salts ${ }^{23-25}$. Meanwhile, the other commonly used fluoride agent SMFP has great compatibility with calcium containing abrasives due to the fluorophosphate $\left(\mathrm{PO}_{3} \mathrm{~F}_{2}^{-}\right)$component in $\mathrm{SMFP}^{23}$. The firm bond between the fluoride ion and phosphate in the $\mathrm{PO}_{3} \mathrm{~F}_{2}^{-}$inhibits the $\mathrm{PO}_{3} \mathrm{~F}_{2}^{-}$from binding to calci$u^{23}$. In this study, The toothpaste 4 and the toothpaste 5 had $\mathrm{NaF}$ as their fluoride agents and silica as their abrasives, and the toothpaste 6 contained KF, which is another ionic compound like NaF, and calcium glycerophosphate as abrasive. Although NaF is known to be incompatible with calcium containing abrasive, its formulation with silica allows fluoride to be soluble $e^{24,26,27)}$, which can also be supported by the present study's finding with the toothpaste 5 . Its measured concentration was $92.48 \%$ of the declared, showing the second highest similarity to the declared concentration among the six toothpastes (Fig. 1). Moreover, a previous study reported that this ionic compound is compatible calcium phosphate abrasive ${ }^{222}$, which explains the highest fluoride release of the toothpaste 6. The toothpaste 6 had the highest similarity percentage between the measured and the declared concentration with the similarity value of $93.31 \%$. In addition to the formulation compatibility, the toothpaste 6 is in gel form, which could also have attributed to its highest fluoride release. Meanwhile, the toothpaste 4 had relatively low similarity percentage between the measured and the declared concentration although of its formulation between NaF and silica. This might be due to other components within the toothpaste, hampering the complete dissolution of the toothpaste.

Regarding the measured TF concentrations of the toothpastes containing non-ionic fluoride compounds, it was found that less fluoride was soluble from non-ionic fluoride toothpastes than the ionic fluoride toothpastes. The measured concentrations of the toothpaste 1, which contained SMFP as fluoride agent and DCPD as abrasive, and the toothpaste 2, which was formulated of AmF and silica, were $74.49 \%$ and $53.48 \%$ of the declared concentrations. The toothpaste 2 had the largest difference between the declared and the measured TF concentrations, showing almost a half similarity when compared to the toothpaste 6 . This may be due to the poor compatibility between the abrasive and the fluoride agent. However, there was a previous study that compared the uptake of fluoride by tooth enamel from four different fluoride toothpastes: sodium fluoride (NaF), sodium monofluorophosphate (NaMFP), stannous fluoride $\left(\mathrm{SnF}_{2}\right)$, and amine fluoride $(\mathrm{AmF})$, and found that the uptake of fluoride was statistically significant in groups of $\mathrm{AmF}$ and $\mathrm{SnF}_{2}{ }^{28}$. Thus, although the concentration of fluoride was low, the actual uptake might be high, affecting the effectiveness of fluoride on caries.

In this study, the six toothpastes were also measured of their 1-minute potentially bioavailable fluoride. Although the recommended brushing time is 2 or 3 minutes, a majority of studies indicated the average brushing time to be approximately 1 minute ${ }^{14,15)}$. Moreover, the ADA guidelines for fluoride-containing dentifrice products also describes the measurement method for one minute fluoride release rate in fresh and aged samples, emphasizing the importance of measuring the bioavailable fluoride ${ }^{29}$. In order to simulate this actual situation, the present study mixed toothpaste slurries for 1 minute, centrifuged them for 2 minutes, ionized them with acid, and obtained the bioavailable fluoride concentrations. As observed in the TF measurement, the BF concentrations of all six toothpastes were lower than the declared TF concentrations with various differences. For the BF measurement, the six toothpastes could be classified into two groups, the clear group and the opaque group, according to the status of samples after the centrifuge, and only two toothpastes 5 and 6 were included in the clear group. When the BF concentration of the toothpaste 6 was compared to the declared fluorides and the measured TF, it presented the highest similarity. However, the $\mathrm{BF}$ concentration of the toothpaste 5 was $75.80 \%$ of the expected fluoride and $82.18 \%$ of the measured TF, indicating the advantage of the gel property of the toothpaste 6 in terms of releasing bioavailable fluoride (Fig. 1). Meanwhile, the four toothpastes of the opaque group presented different patterns from the clear group. The toothpaste 2 and 4 showed their BF concentrations much higher than the measured TF concentrations; whereas the BF concentrations of the toothpaste 1 and 3 were lower. This may be due to the fact that although all toothpaste samples were discarded unless they were completely suspended into the slurry, 1 minute was still too short to completely disperse the toothpastes, resulting in insoluble salt included in the supernatants. Therefore, there is a probability that the BF concentrations of the four toothpastes measured with the newly suggested measurement method might have been overestimated. Furthermore, as surfactants and binder agents were reported to inhibit salivary and plaque phosphatases by a previous study ${ }^{30)}$, there is a chance that formulation components other than abrasives could also have affected bioavailability of fluoride ions. Hence, there is a need to review the newly suggested measurement method protocols, where none of salivary and plaque phosphatases was considered. 
According to Cochrane review, it was stated that, for preventing caries in children and adolescents, toothpastes must contain at least 1,000 ppm fluoride ${ }^{5}$. In Korea, fortunately, there was a recent amendment to the legislation in which the maximum permitted fluoride content in toothpaste was increased from 1,000 ppm to 1,500 ppm. From this change, there will be more bioavailable fluoride ions delivered by the toothpastes in Korea, increasing the effectiveness of Korean toothpastes.

The concern of quality of fluoride toothpaste is not only restricted to Korea as international guidelines for toothpastes often refer to only the total fluoride content, and the ISO Standard 11609 was not an exception. Nevertheless, recently there was a new suggestion by ISO about measuring the bioavailable fluoride, and the present study analyzed six toothpastes of their total and 1minute potentially bioavailable fluoride concentrations according to the proposed method. Although the-1 minute bioavailable fluoride measurement method could be considered as the significant method as it was designed to mimic the actual brushing situation, the method seemed to be affected by various factors including compatibility between fluorides and abrasives. Therefore, the newly suggested method requires further revision, which would lead to development of a useful measurement method to assess fluoride bioavailability in toothpastes.

\section{Conclusions}

Six toothpastes were analyzed of total and 1-minute potentially bioavailable fluoride concentrations according to newly suggested ISO method, and both total and bioavailable concentrations were compared with the fluoride concentration written on the packaging.

When compared to the declared fluoride concentrations, deficiencies were found regarding both total and bioavailable fluoride concentrations for all the six toothpastes. This leads to a need to monitor the quality of fluoride toothpastes on a regular basis.

In particular, the results of bioavailable fluoride concentration measurements were hugely affected by the compositions and properties of toothpastes.

Since the measurement results of bioavailable fluoride concentrations obtained using the newly suggested method seem to be inadequate to be interpreted as representative bioavailable fluoride, it is necessary to revise the method in order to guarantee a greater standardization.

\section{References}

1. Glass RL. First International Conference on the Declining Prevalence of Dental Caries. J Dent Res 1982;61:1304-1383.

2. Petersen PE, Lennon MA. Effective use of fluorides for the prevention of dental caries in the 21st century: the WHO approach. Community Dent Oral Epidemiol 2004;32:319-321.

3. Ripa LW. A critique of topical fluoride methods (dentifrices, mouthrinses, operator-, and self-applied gels) in an era of decreased caries and increased fluorosis prevalence. J Public Health Dent 1991; 51:23-41.

4. Glass RL. Caries reduction by a dentifrice containing sodium monofluorophosphate in a calcium carbonate base. Partial explanation for diminishing caries prevalence. Clin Prev Dent 1981;3:6-8.

5. Marinho VC, Higgins JP, Sheiham A, Logan S. Fluoride toothpastes for preventing dental caries in children and adolescents. Cochrane Database Syst Rev 2003:CD002278.

6. Wong MC, Clarkson J, Glenny AM, Lo EC, Marinho VC, Tsang BW, et al. Cochrane reviews on the benefits/risks of fluoride toothpastes. J Dent Res 2011;90:573-579.

7. Bibby BG. A test of the effect of fluoride-containing dentifrices on dental caries. J Dent Res 1945;24:297-303.

8. Kyes FM, Overton NJ, McKean TW. Clinical trials of caries inhibitory dentifrices. J Am Dent Assoc 1961;63:189-193.

9. Ericsson Y. Fluorides in dentifrices. Investigations using radioactive fluorine. Acta Odontol Scand 1961;19:41-77.

10. van Loveren C, Moorer WR, Buijs MJ, van Palenstein Helderman WH. Total and free fluoride in toothpastes from some non-established market economy countries. Caries Res 2005;39:224-230.

11. Kim J-H, Jeong S-S, Choi C-H, Hong S-J. Fluoride concentration of commercial dentifrices and effect of fluoride containing dentifrices on artificial enamel caries. Journal of Korean academy of oral health 2006;30:56-66.

12. Hong S-J, Park Y-N, Jeong S-S, Ha M-O, Choi C-H, Lee K-H. Fluoride concentration of commercial dentifrices for children in Korea. Journal of Korean academy of oral health 2008;32:143-151.

13. ISO. ISO 11609:2010 Dentistry-Dentifrices-Requirements, test methods and marking. Geneva:ISO;2010.

14. Lefkoff MH, Beck FM, Horton JE. The effectiveness of a disposable tooth cleansing device on plaque. J Periodontol 1995;66:218-221.

15. Keyes PH. The infectious and transmissible nature of experimental dental caries. Findings and implications. Arch Oral Biol 1960;1:304320.

16. Tenuta LM, Cury JA. Fluoride: its role in dentistry. Braz Oral Res 2010;24 Suppl 1:9-17.

17. Carrera CA, Giacaman RA, Munoz-Sandoval C, Cury JA. Total and soluble fluoride content in commercial dentifrices in Chile. Acta Odontol Scand 2012;70:583-588.

18. White DJ. Reactivity of fluoride dentifrices with artificial caries. III. Quantitative aspects of acquired acid resistance (AAR): F uptake, retention, surface hardening and remineralization. J Clin Dent 1991; 3:6-14.

19. Hattab FN. Analytical methods for the determination of various forms of fluoride in toothpastes. J Dent 1989;17:77-83.

20. Gleisner H, Einax JW, Mores S, Welz B, Carasek E. A fast and accurate method for the determination of total and soluble fluorine in toothpaste using high-resolution graphite furnace molecular absorption spectrometry and its comparison with established techniques. J Pharm Biomed Anal 2011;54:1040-1046.

21. Biemer TA, Asral N, Sippy A. Ion chromatographic procedures for analysis of total fluoride content in dentifrices. Journal of Chromatography A 1997;771:355-359.

22. Pessan JP, Toumba KJ, Buzalaf MA. Topical use of fluorides for car- 
ies control. Monogr Oral Sci 2011;22:115-132.

23. Benzian H, Holmgren C, Buijs M, van Loveren C, van der Weijden F, van Palenstein Helderman W. Total and free available fluoride in toothpastes in Brunei, Cambodia, Laos, the Netherlands and Suriname. Int Dent J 2012;62:213-221.

24. Conde NC, Rebelo MA, Cury JA. Evaluation of the fluoride stability of dentifrices sold in Manaus, AM, Brazil. Pesqui Odontol Bras 2003; 17:247-253.

25. Stookey GK, DePaola PF, Featherstone JD, Fejerskov O, Moller IJ, Rotberg S, et al. A critical review of the relative anticaries efficacy of sodium fluoride and sodium monofluorophosphate dentifrices. Caries Res 1993;27:337-360.
26. de Freitas JF. Fluoride stability in toothpastes. Aust Dent J 1984;29:30-35.

27. Hattab FN. The state of fluorides in toothpastes. J Dent 1989;17:4754.

28. Patil VH, Anegundi RT. An in vitro assessment of fluoride uptake by tooth enamel from four different fluoride dentifrices. Eur Arch Paediatr Dent 2014.

29. ADA. Acceptance Program Guidelines: fluoride-containing dentifrices. Chicago:Council on Scientific Affairs;2005.

30. Gaffar A, Polefka T, Afflitto J, Esposito A, Smith S. In vitro evaluations of pyrophosphate/copolymer/NaF as an anticalculus agent. Compend Suppl 1987:S242-250. 